\title{
Enhanced Mechanical Calibration of Dissolution Test Equipment
}

\author{
Alger Salt ${ }^{1, *}$ and John Glennon ${ }^{2}$ \\ GlaxoSmithKline, Product Development Laboratory Automation and Platforms, \\ 5 Moore Drive, Research Triangle Park, NC 27709 \\ ${ }^{2}$ GlaxoSmithKline, Global Manufacturing and Supply, Shared Stability Services 1011 \\ N. Arendell Ave., Zebulon, NC 27597
}

e-mail:alger.d.salt@gsk.com

\section{INTRODUCTION}

n late 2009 the GSK R\&D Product Development organization began implementing a new approach to calibration of dissolution test equipment, specifically Apparatus 1 and 2 (baskets and paddles). The new approach, enhanced mechanical calibration (EMC), which was recommended by FDA, follows the ASTM International Standard Procedure ASTM 2503-07 (1). This article explains why this change was made, what was done to effect the change, how it was accomplished, and things we learned in the process.

\section{REASONS FOR CHANGE}

A key driver for implementing this change was to enhance confidence in results produced by the dissolution test equipment. We believed that the more stringent requirements imposed by the EMC approach would result in better control of our instruments. We had long suffered issues using the USP Performance Verification Test (PVT) as a means to qualify dissolution testers that included:

- Oversensitivity of Prednisone Tablet Reference Standards to dissolved gas in the medium.

- Very broad specifications that are not representative of those normally applied to our products.

- Change in the specification, sometimes due to changes in the particular lot of tablets.

We believed that the PVT was more of a test of analyst or technician proficiency than of performance attributes of the instruments under test. If a PVT failed, it was often difficult to determine the root cause of the failure. Typically, tests were simply repeated with more attention to media preparation, tablet introduction, or collection of the dissolution sample solutions; attention to these details almost always resulted in passing results.

Besides scientific reasons, there were business reasons for the change. About one full day per system was required to perform PVT, which includes media preparation, test performance, and results documentation. Initial estimates indicated that a procedure based on the EMC approach would require about half the time as the USP approach. In addition, the EMC approach obviates the need for PVT and associated reference standard materials, further reducing costs.

${ }^{*}$ Corresponding author.

\section{FINANCIAL ANALYSIS}

We started planning for a transition from the USP-based to the EMC-based qualification procedures in 2007, with the first round of enhanced mechanical calibrations conducted in 4Q09. The transition took about six months to complete. This initiative was global in scope, covering dissolution equipment at three different R\&D sites in North America and four R\&D sites in Europe.

We estimated that the time required to perform USP calibrations was about ten hours per instrument or twenty hours per year based on a six-month calibration interval. Note that the USP procedure also incorporates testing the mechanical attributes and dimensional measurements, which consumes about half of the total time per system. We projected that this time would be reduced to around six hours per event or twelve hours per year to perform enhanced mechanical calibrations. Additional savings would be realized by not maintaining stocks of Prednisone Tablet Reference Standards at each site. It was determined that some initial investment would be required to make this change. These investments would include expenses associated with replacing or certifying a large portion of our inventory of dissolution vessels (\$50-100 each). New versions of the measurement tools were needed to perform some of the ASTM tests such as vessel verticality. We also had to purchase specialized measurement tools needed for specific brands of dissolution test equipment. These one-time capital investments totaled to around $\$ 12 \mathrm{~K}$ at each site.

Figure 1 is a compilation of the financial analysis done in 2007 that covered a period of six quarters beginning in 2008. (In reality, the time axis on this chart should be shifted forward by five quarters due to some unanticipated delays in implementing the change.) The one-time capital costs were not amortized. A recent review of current costs confirmed that our estimates were on target. Switching to the EMC approach provided a rapid return on investment.

\section{RISK EVALUATION}

As part of the transition process, a risk assessment was performed on the variable factors that are part of the EMC process and that could influence the performance of a dissolution test. It should be noted that the risk assessment that will be described is based on the opinions 


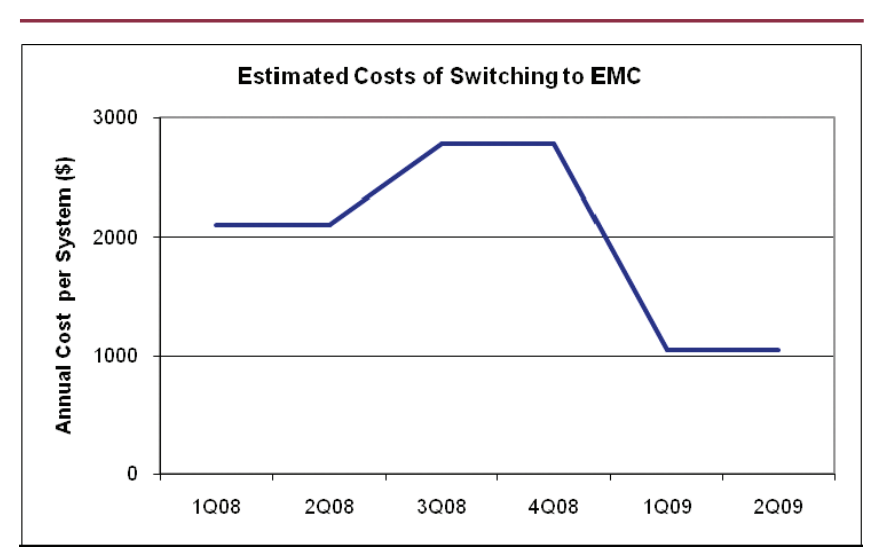

Figure 1. Financial analysis of EMC benefits.

of the GSK subject matter experts involved at the time it was performed. As such, it formed the basis for the GSK R\&D move to EMC. The reader may not agree with the classifications and risk ratings. Indeed, the variable classification and risk assessment may be different from company to company, or even from lab to lab. The main intent here is to describe the process of performing the risk assessment.

The initial step was to classify the different variables into three groups, controlled variables, experimental variables, and noise variables. Controlled variables are those that have controls already defined for them. Experimental variables require some form of experimental testing to ascertain their impact. Noise variables have an amount of uncertainty as to their effect on the EMC process. Table 1 shows all variables considered and their classification.

No specific risk assessment was made on variables deemed to be controlled variables. The experimental variables were reviewed in terms of their effect on the process and prioritized among themselves, because all were considered not to have a specific effect on the calibration of a dissolution system but rather on actual testing performed with a system. All variables classified as noise variables were evaluated using a failure mode and effect analysis (FMEA). This involved determining how severe a problem with the cited variable would be, how likely it is to happen, and the likelihood that the problem would be detected. Table 2 shows the results of the FMEA.

As can be seen in Table 2, none of the noise variables was considered high risk, and the vast majority of them were low risk. Most of the medium-risk items depend on the condition of the parts of the system. This indicates that it is imperative for those performing the EMC to pay particular attention to that aspect. However, one part of the ASTM 2503-07 requirements elevated what was assessed as a low-risk variable (i.e., the dimensions and shape of the vessels used) to a more significant issue. Section 4.3.1, Vessel Dimensions, of ASTM 2503-07 (1) states, "In the absence of a COA or COC, the vessel's
Table 1. Classification of Variables Involved in EMC

\begin{tabular}{|c|c|}
\hline Variable & Variable Class \\
\hline Water Bath condition & Controlled \\
\hline Water bath Temperature Stability & Controlled \\
\hline Material for Basket Shaft & Controlled \\
\hline Dimensions for Basket Shaft & Controlled \\
\hline Materials for Baskets & Controlled \\
\hline Dimensions for Paddle Shaft & Controlled \\
\hline Dimensions for Paddles & Controlled \\
\hline $\begin{array}{l}\text { Instructions on Performing Mechanical } \\
\text { Calibration }\end{array}$ & Controlled \\
\hline Compliance with USP, EP,JP, etc. & Controlled \\
\hline Materials for Paddle Shaft & Experimental \\
\hline Material for Paddles & Experimental \\
\hline $\begin{array}{l}\text { Basket Holding mechanism (clips or } \\
\text { o-rings) }\end{array}$ & Experimental \\
\hline Basket Wobble & Noise \\
\hline $\begin{array}{l}\text { Condition of Baskets } \\
\text { (not deformed, etc.) }\end{array}$ & Noise \\
\hline $\begin{array}{l}\text { Condition of Vessels (smooth surfaces, } \\
\text { no chips, etc.) }\end{array}$ & Noise \\
\hline Manufacturer & Noise \\
\hline Materials for Vessels & Noise \\
\hline Condition of Paddles & Noise \\
\hline Vessel Centering & Noise \\
\hline Vessel Temperature & Noise \\
\hline Shaft wobble & Noise \\
\hline Vessel Verticality & Noise \\
\hline Rotational Speed & Noise \\
\hline Shaft Verticality & Noise \\
\hline Basket and Paddle Depth & Noise \\
\hline Dimensions/Shape for Vessels & Noise \\
\hline Dimensions for Baskets & Noise \\
\hline
\end{tabular}

internal dimensions should be measured with an appropriate measuring device."The imperative from the ASTM procedure is that the dimensions of each vessel must be known. Because ASTM 2503-07 gives no specific dimensions or tolerances for vessels, those in the harmonized dissolution General Chapters of the USP, European Pharmacopoeia (EP), and the Japanese Pharmacopoeia (JP) were used. Given the large number of vessels within the 


\begin{tabular}{|c|c|c|}
\hline Variable & Variable Class & $\begin{array}{c}\text { FMEA } \\
\text { Risk Score (L/M/H) }\end{array}$ \\
\hline Basket Wobble & Noise & Medium \\
\hline $\begin{array}{l}\text { Condition of Baskets } \\
\text { (not deformed, etc.) }\end{array}$ & Noise & Medium \\
\hline $\begin{array}{l}\text { Condition of Vessels } \\
\text { (smooth surfaces, no } \\
\text { chips, etc.) }\end{array}$ & Noise & Medium \\
\hline Manufacturer & Noise & Medium \\
\hline Materials for Vessels & Noise & Medium \\
\hline Condition of Paddles & Noise & Medium \\
\hline Vessel Centering & Noise & Low \\
\hline Vessel Temperature & Noise & Low \\
\hline Shaft wobble & Noise & Low \\
\hline Vessel Verticality & Noise & Low \\
\hline Rotational Speed & Noise & Low \\
\hline Shaft Verticality & Noise & Low \\
\hline $\begin{array}{l}\text { Basket and Paddle } \\
\text { Depth }\end{array}$ & Noise & Low \\
\hline $\begin{array}{l}\text { Dimensions/Shape for } \\
\text { Vessels }\end{array}$ & Noise & Low \\
\hline Dimensions for Baskets & Noise & Low \\
\hline
\end{tabular}

GSK R\&D organization, it was unlikely that a COA or COC was available for all of them, and the approach to taking such measurements accurately was seen as a challenge. Consequently, as part of the introduction of EMC, a program was developed to obtain the dimensions of all vessels that did not have a COA or COC using three-dimensional coordinate measurements. This did not imply that the vessels in use were not compliant with the pharmacopeial dimension requirements, but rather provided direct evidence of their compliance.

\section{COMPLIANCE ASPECTS}

One of the major challenges in implementing EMC was the compliance aspect of not following the procedures described in USP <711 > Dissolution. Although USP <711> is a harmonized chapter aligned with the $E P$ and the $J P$, the USP Performance Verification Test (PVT), or the Apparatus Suitability Test as it was known before 01 August 2007, is a requirement for USP only.

ASTM 2503-07 was issued by ASTM International in April 2007 (1). The main reason for the introduction of this standard was the recognition that USP Apparatus Suitability Test using USP calibrator tablets at that time was not truly "calibrating" dissolution units, but rather

checking the performance of the systems. This distinction arose from the fact that when a failure occurred, it was usually the result of an analytical issue (frequently improper degassing) rather than an issue that could be resolved by adjustment of the apparatus.

In the absence of any specific guidance from the FDA at the time, compliance concerns were eased by the source of the ASTM initiative. The ASTM standard was based on the results of investigations into the variability of USP calibrator tablets, particularly prednisone, performed by the FDA and the subsequent procedures put in place for the calibration of dissolution systems in FDA laboratories (2). The outcome of this work was that appropriate control of the mechanical tolerances of a dissolution unit could reduce the variability in the test and effectively leave the tablets as the only significant source of variability.

ASTM 2503-07 sets requirements for all the mechanical tolerances specified in the harmonized pharmacopeial monograph and includes several additional requirements (e.g., dual-point vessel centering, shaft verticality, and requirements for vessel temperature sensors). All the requirements of the ASTM standard are the same as or tighter than those in the pharmacopeia (USP,EP, and JP).

The compliance aspect became much clearer in January 2010 when the FDA released its official guidance on mechanical calibration of dissolution Apparatus 1 and 2 (3), which states, "We recommend that an appropriately enhanced procedure for MC (Mechanical Calibration) can be applied to USP Dissolution Apparatus 1 and 2 as an alternative procedure to meet CGMP calibration requirements." ASTM E2503-07 is identified in the guidance as a suitably rigorous calibration method. A similar statement is made in the FDA Considerations for Implementation section of ICH Q4B Evaluation and Recommendation of Pharmacopoeial Texts for Use in the ICH Regions, Annex 7, Dissolution Test General Chapter. The FDA guidance further states, "Calibration procedures that rely solely on tests using reference standard tablets are generally not recommended, since they do not provide assurance that the apparatus is adequately calibrated, nor provide a reliable basis upon which to make precise tolerance adjustments to the dissolution apparatus."

Therefore, from a United States regulatory compliance perspective, the use of EMC in and of itself should not pose any regulatory risk. It is recognized, however, that regulatory authorities in other markets employ the USP as a standard. For supplying such markets, it is prudent to evaluate any potential compliance risks that EMC would present given that it does not follow the USP verbatim.

\section{CHANGE MANAGEMENT AND IMPLEMENTATION}

To prepare for the change in calibration procedures, a technical memorandum was issued to document formally the intent to change calibration procedures and the rationale for the change. It was, in effect, a high-level change-control plan. It included the reasons for change, the impact, and the implementation schedule. It also 
included a provision for some sites to remain with the traditional USP-based test procedures during the transition.

A key part of the implementation of the EMC was defining the process for the transition from USP PVT to EMC. This became evident when several systems that had passed PVT at their previous evaluation experienced an initial failure using EMC. This provided a challenge with regard to assuring that data generated on these systems during the six months before EMC testing were not impacted. The challenge was met by performing the PVT to "close out" the six months, identifying the root cause of the EMC failure, resolving it, and then re-performing the EMC. Because of this experience, the process for the transition from PVT to EMC has been defined as a one-time exercise consisting of:

1. Performing the PVT.This "closes out" the PVT era for the unit.

2. Performing the EMC. This starts the EMC era for the unit.

Enhanced Mechanical Calibrations revealed some specific issues that had previously not been detected by the former USP-based tests. The first site to implement the new procedures encountered a high number of EMC failures for vessel centering. Interestingly, the root cause for the failures was determined to be the vessel dimensions. The units that had issues used less expensive vessels purchased through a third-party supplier rather than those from the unit manufacturer. These systems met the USP PVT requirements for several years but did not meet the additional acceptance criteria associated with the EMC-based procedures for vessel centering. Once the third-party vessels were replaced with certified, serialized vessels supplied by the original equipment vendor, the systems passed the EMC-based test procedures. This experience validated the conclusions of the risk analysis that had been performed and further bolstered our confidence that we had made the right decision in switching to the new approach.

\section{VESSELS}

As stated before, dissolution vessels were identified as the most "risky" of the removable components of the dissolution testers because confirming the dimensions of the vessels presented a significant challenge. To mitigate this risk, all vessels were uniquely identified with a serial number, and each vessel was certified (either by the vendor or by independent measurements) to comply with the dimensions specified in the USP, EP, and $J P$.

Within R\&D, we possessed a significant inventory of non-serialized vessels. Replacing this inventory would have been very expensive. Metrology Solutions, Inc. (Romeoville, IL) provided three-dimensional coordinate measurements that allowed us to certify that the physical dimensions of each vessel complied with the USP,EP, and $J P$ requirements. The cost for this service was less than half the price of purchasing new vessels. If vessels did not already have a serial number, each one was serialized with a unique identifier before shipping for measurement. Each vessel was returned with a certificate listing the actual dimensions.

Before using EMC, we did not pay particular attention to the positioning of vessels and removable components. We assumed that all parts were interchangeable. Because of this change, all removable components (vessels, shafts, paddles, etc.) are serialized and returned to their original positions whenever they are removed. Typically, the removable components are installed via rank-order of their serial number.

\section{AFTER-ACTION REVIEW}

The implementation team participated in an afteraction review. We believed that our manufacturing site laboratories could benefit from our learning when they decide to implement a similar change in their qualification process. The output from the after-action review is as follows:

- As part of the switch to ASTM mechanical calibrations, perform the USP PVT to establish an as-found status of each instrument. Perform as-found mechanical calibrations and adjust if necessary to meet acceptance criteria.

- Be clear from the beginning about what to do in the event of failures.

- Get existing vessels certified or obtain certified vessels from vendor(s) in advance.

- Alert the user community to all differences in how they should handle the equipment, specifically regarding replacing vessels and other removable components. Some dissolution testers require specific rotational orientations to meet the specification for vessel centering.

- Anticipate the need and budget for additional calibration tools and instruments.

- Mechanical calibration is MORE rigorous and discerning than USP PVT.

- Consider a formal risk analysis (what could go wrong, what is the severity if it did, would we know it had gone wrong).

- The quality of the dissolution vessel is critical. Having spare vessels is crucial.

- The rotational orientation of the vessels may affect the measurement for vessel centering. It may be necessary to mark the vessels and the top plate of the dissolution tester to maintain this rotational orientation.

\section{CONCLUSION}

All dissolution apparatus used within GSK R\&D Product Development functions globally are now calibrated using EMC. As a result, there is now tighter control over the performance of the dissolution kit, which provides a greater assurance of the quality of dissolution data 
generated. In addition, significant savings in terms of both time and money for calibrations have been realized; hence, we are increasing quality while controlling costs. We believe that the new approach to qualifying Apparatus 1 and 2 dissolution testers that incorporates EMC is superior to the traditionally applied USP PVT approach, and that belief has been ratified by our experiences. Going forward, it is intended to expand the use of EMC into the GSK manufacturing quality laboratories.

\section{REFERENCES}

1. Standard Practice for Qualification of Basket and Paddle Dissolution Apparatus; ASTM Standard E2503-07; ASTM International:West Conshohocken, PA, 2007.
2. Mechanical Qualification of Dissolution Apparatus 1 and 2; DPA-LOP.002; U.S. Department of Health and Human Services, Food and Drug Administration, Division of Pharmaceutical Analysis, U.S. Government Printing Office:Washington, DC, 2006. http://www.fda.gov/ downloads/AboutFDA/CentersOffices/CDER/ UCM142492.pdf (accessed Apr 10, 2011).

3. The Use of Mechanical Calibration of Dissolution Apparatus 1 and 2-Current Good Manufacturing Practice (CGMP); Guidance for Industry; U.S. Department of Health and Human Services, Food and Drug Administration, Center for Drug Evaluation and Research (CDER), U.S. Government Printing Office: Washington, DC, January, 2010. 